\title{
INTEGRATING HUMAN FACTORS IN THE DESIGN OF SAFETY CRITICAL SYSTEMS
}

\author{
A barrier based approach
}

\author{
Bastiaan A. Schupp ${ }^{1}$, Shamus P. Smith ${ }^{1}$, Peter C. Wright ${ }^{1}$, Louis H.J. Goossens ${ }^{2}$ \\ ${ }^{1}$ University of York, Department of Computer Science, Heslington, York, YO10 5DD, United \\ Kingdom, \{ bastiaan.schupp / shamus.smith / peter.wright \}@cs.york.ac.uk
}

${ }^{2}$ Delft University of Technology, Department of Technology, Policy and Management, Safety Science Group, Jaffalaan 52828 BX Delft, the Netherlands, l.h.j.goossens@tbm.tudelft.nl

\begin{abstract}
Human factors contribute to risk in safety critical systems. However, current approaches to integrating human factors issues in the development of safety critical systems appear not fully sufficient. In this paper a new approach is proposed based on a technique from chemical engineering risk analysis called Safety Modelling Language (SML). SML provides a way to conceptually design risk reduction based on barriers. The approach further helps to design and implement safety barriers. The approach is demonstrated using a case in which human factors play an important role from the medical domain.
\end{abstract}

Keywords: Human Factors, Design, Safety, Barriers, Methods, Risk, Risk Reduction

\section{INTRODUCTION}

Risk reduction is a key factor in the design of safety critical systems. Human factors are an essential part of the risk reduction process. When systems become operational the human may either create accidents, or help as part of the system to prevent them. The integration of human factors analysis into systems design is traditionally a difficult problem (Hollnagel 1993). Designers need to be able to explore and evaluate solutions. In this paper an approach is presented that allows the design of risk reduction and safety barriers and supports barrier implementation. 
Traditionally risk reduction is dealt with late in the system design process when all details are clear, and economic and safety benefits can be gained from designing risk reduction from the design onset (Arthur D. Little 2001, Schupp et al. 2002). Usually, at an early stage, multiple options exist to achieve the most optimal design. However if risk reduction is considered too late in the design process options are lost as changing earlier design decisions becomes prohibitively expensive. Hence, designers must have the ability to identify potential problems, to estimate the risk, and to find and evaluate solutions early in design.

The format for the remainder of this paper is as follows: Section 2 outlines our approach and briefly describes its main components, (i) the barrier concept, (ii) Safety Modelling Language (SML) and (iii) the mapping between SML and a design. Section 3 presents the case study of this paper. It is a case from the medical domain that deals with human factor issues in the design of a computer assisted detection system for mammography. Section 4 presents concluding remarks.

\section{INTEGRATING HUMAN FACTORS IN DESIGN}

When a system becomes operational its properties may create adverse effects, i.e. hazards, to the system itself, or to its environment. A design team will use tools to identify such potential hazards. If humans participate in the system, the designers may resort to well established human factors methods such as human reliability analysis (HRA), THERP (Kirwan 1994), or HEART (Williams 1986), or to human factor experts.

After identifying issues that lead to unacceptable risks, the designers may either decide to change the design to prevent hazards or to add barriers that protect the target. Barriers are designed in a similar manner as the initial system. A barrier may also have adverse effects and when it fails, it will no longer prevent or protect against the hazard it was designed for. Hence the designers of the barrier analyse it in a similar manner as the initial system. This process is complex, as the barrier may be implemented across multiple system components.

One of the obstacles to integration of human factors analysis into conceptual system design is the lack of a suitably expressive language to represent and analyse safety at a conceptual level. Most existing human factor methods are for identification or quantification, and do not help in finding solutions. Similar observations are made by Swuste (Swuste 1996) and Harms-Ringdahl (Harms-Ringdahl 2003). Hence, our method is solution oriented and provides a framework for designers to explore and evaluate solutions. This is based on the previously developed SML (Schupp et al. 
2001), which aids designers in conceptually designing risk reduction. It was developed for use in the chemical process domain, but we will show that it can be used in other domains as well, and how to integrate human factors into it.

The proposed method deals with the design of two systems: The safety critical system itself, and the barrier systems that mitigate its risks. As barriers are important in risk reduction these are the main building block of our method. The explicit but conceptual representation of barriers which we describe in this paper translates the results of human factors methods into a design representation to create an overview of how barriers will become implemented and how risk reduction is achieved. Usually it is not the original designers of the safety critical part of the system that are involved in designing, implementing, maintaining and operating barriers, but other actors. Hence, by creating overview, the method also helps to disseminate information to these actors.

\subsection{Barriers}

The word barrier is commonly used in normal language as well as in risk management and human factors domains. However, A well accepted 'barrier theory' does not exist. Nevertheless it has been shown that barriers can be a viable way to study human factors (Kecklund et al. 1996), to analyze systems (Johnson 1980), and there exists attempts to classify barriers by their physical implementation e.g. (American Institute of Chemical Engineers 1993) and (Hollnagel 1999). Sometimes barriers are named differently, for instance layers of protection (Dowell 1998). Our own notion of barriers is based on Haddon's (Haddon 1973) fundamental strategies for risk reduction which culminates in the hazard barrier target model (described in Section 2.2).

Here barriers are always considered as systems as they almost always have multiple components. We define barriers as the combination of technical, human and organisational measures that prevent or protect against an adverse effect. A typical barrier may have three components, one to detect, one to decide, and one to deflect. An example of a barrier is a nonsmoking sign. However the barrier is not the sign as such; it includes awareness of how smoking may cause fire, awareness of the significance of the sign, its state and location, its maintenance, training of the smokers, and its relation to other barrier systems. If a non-smoking sign is put in the wrong place, it will not work. Hence the location is part of the barrier. Similarly, a brake is not a barrier but the actuator in a braking system also involving detection and decision. Its function is not to brake, but is part of a system by which, for instance, collisions are avoided. Here our approach 
differs from some other sources in literature where the sign or the brake are considered to be the barrier, for instance Hollnagel (Hollnagel 1999).

Humans interact closely with barriers. Obviously barriers may protect humans, but humans can be part of barriers, can make them fail, and must maintain them.

Partly based on a classification made by Swuste (Swuste 1996), we study barriers here at three levels:

- At the safety function level; this concerns the role of the barrier in system safety. For example, the role of the non-smoking barrier in the greater context of preventing fire in a building. At this level barriers are considered black boxes, i.e. the internal structure and functions are ignored.

- At the barrier form level; this concerns how a barrier functions and what its components are, thus what is inside the black box introduced at the safety function level. For example, the components introduced when discussing the non-smoking sign; the sign, training, and maintenance. This level demonstrates which functions the system should provide to allow the barrier to function.

- At the embodiment level; this concerns the detailed design of the barrier, and its physical representation and implementation in the safety critical system. For example, the requirement that a non-smoking sign with specified size should be placed at a specified position on every access door.

These three levels are the core of our method. We use SML at the safety function level to design an optimal risk reduction strategy, based on information from the form level and the embodiment level. At the form level the basic design and analysis of barriers takes place, while the whole of the safety critical system and the implementation of the barriers therein are addressed at the embodiment level.

\subsection{The Safety Modelling Language}

SML provides a means to design and document the function of barriers in a system. It provides a framework that allows a designer to define the problem, to analyze knowledge for solving the problem, to synthesize possible solutions, and to analyze the performance of these solutions. Furthermore, it helps the designer to communicate. A very important use of SML not discussed in this paper is that its relational structure offers a efficient means for storing information, for example for storing experience about barrier performance, for later reuse. The language can be used during all life-cycle stages of a system, thus including all design stages, implementation and operation. 
SML is based on the Hazard-Barrier-Target (H-B-T) model (Schupp et al. 2001), which assumes that targets are vulnerable to the effects of hazards. In some respects it is similar to other barrier models, such as the accident evolution and barrier function model (Svenson 1991), and the 'Swisscheese' model (Reason 1990). However, its main focus is on design and communication, not on analysis. The main means of communication in the SML are diagrams, as in Figure 1. This diagram shows how toxic fumes are hazardous to workers, as these poison them. However the worker is protected by a containment system that contains the fumes, thus being a barrier that prevents exposure. As this may not be completely adequate, the worker is further protected by Personal Protective Equipment (PPE). Alternatively prevention is realized by removing the hazard, for example by using a non-toxic substance.

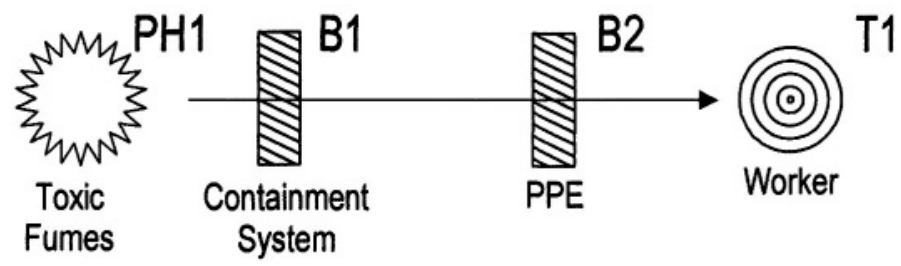

Figure 1. A typical H-B-T diagram. Toxic fumes are hazardous to workers. Hence the workers are protected by a containment system and Personal Protective Equipment (PPE)

The use of SML is relational. A relation between the Hazard and the Target is called an H-T relation. Thus, toxic fumes and workers are an example of an $\mathrm{H}-\mathrm{T}$ relation. This $\mathrm{H}-\mathrm{T}$ relation is itself related to a number of barriers that can act to reduce risk. In Figure 1 these barriers are the containment system and PPE, but may possibly also include barriers such as ventilation, scrubbing, or access procedures. Designers can use the H-T relation to find the optimal barriers for use in their system.

SML models hazards in a more complex manner than the basic H-B-T model in Figure 1. A hazard is something that has the potential to cause an adverse effect to a target. A hazard is a 'label' that humans apply to complex phenomena perceived as hazardous. It is modelled using two components: Causal elements that provide a link to the mechanism of the hazard, and effects, that provide the link to the targets. For instance, when the elements 'flammable substance', 'oxygen', and 'ignition source' are present in a design, these will cause a fire hazard, having heat radiation, smoke and high temperature as effects. This is shown in Figure 2a. A human factors related hazard is a misdiagnosis in interpreting an X-ray photograph in a medical domain. This can for instance be caused by the causal elements 'training', 
'available time', and issues such as 'X-ray clarity'. Effects of a misdiagnosis for instance are false positive or false negative readings (see Figure 2b).

SML does not model the underlying mechanisms of the hazardous phenomena; the modelled hazard is not a direct representation of the mechanism (e.g. physics, or psychology), but it is linked to it by the causal factors. Thus SML does not provide insight into the hazardous phenomenon itself but into the relations this phenomenon has with the rest of the design/system.

Two further aspects of barriers which are relevant to design, but are only briefly discussed in this paper are (i) the types of barriers that can be used, and (ii) modelling how barriers can fail.
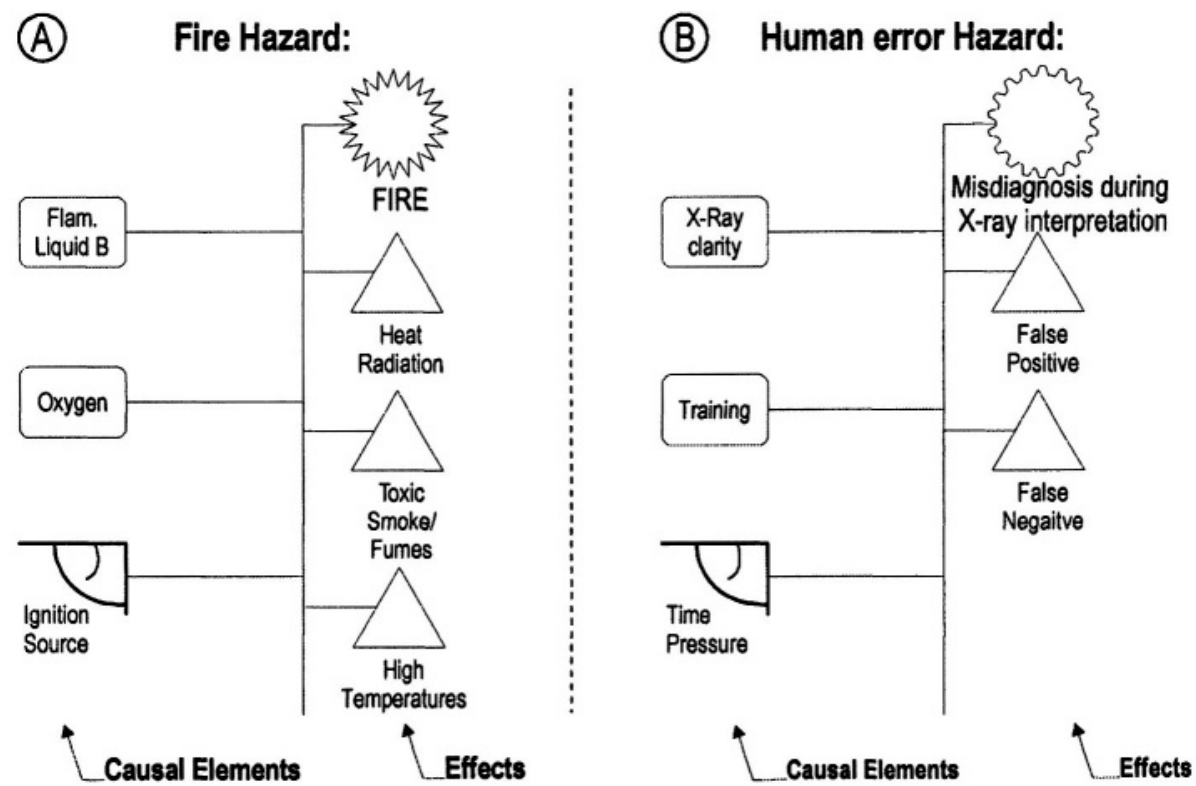

Figure 2. Diagram showing the models of a fire hazard (a), and of a human error hazard (b). The causal elements map to components of the system, while the effects map to targets. The different symbols used for the hazards designate a primary (a) and functional (b) hazard

The types of barriers that are used in SML are classified using three binary dimensions; protective versus mitigative, inherent versus add-on, and preventive versus defensive. This is based on their role in the design, rather then on the nature of the barrier. Thus classifying supports designers in making the role of the barrier clear, without having to worry about the exact form or embodiment of the barriers. The same barrier (e.g. fire protection) may have a different classification in different implementations.

To model the failure of barriers, SML defines primary and functional hazards, the symbols are shown in figure 2. Primary hazards cause direct 
harm to humans, neighbouring installations, and the environment. The barriers in between primary hazards and primary targets are called primary barriers. Functional hazards are phenomena due to either human factors or other causes that adversely affect other barriers, thus making these fail. In this way, a risk reduction problem is defined recursively; when a barrier is used, it can fail due to a functional hazard. It can however be protected by defining functional barriers that protect against this hazard, which can fail as well.

A consequence of this is that the list of primary hazards quickly provides insight in why the systems' safety is critical. Next, accident mechanisms, and the role humans play in these can be understood via recursions. This however is not further explained in this paper.

\subsection{Mapping SML to Form and Embodiment}

To be useful, the SML must be mapped to the form and embodiment level. A detailed discussion of this cannot be provided here. In brief, safety is designed using the SML. Then each barrier is conceptually designed at form level. Subsequently, it is implemented in the design of the overall system at embodiment level. In this paper we represent the conceptual design of the barrier at the form and embodiment level, and of the system using a simplified version of the $\mathrm{SADT}^{31}$ approach. This approach allows hierarchically representing multiple system levels, which is a useful way to study the implementation of barriers. How this occurs is demonstrated in the next section.

\section{MAMMOGRAPHY CASE STUDY}

This section describes a case study originating from the medical domain that illustrates our approach. It involves a socio-technical system in which humans play both a central role as a safety barrier and also are a source of error and potential hazard for the system. The case studies centres on a classic human-factors design decision, of how to combine a level of automation with a level of human intervention to minimise the risk of a system failure.

31 SADT is Structured Analysis and Design Technique, more recently IDEF (not an acronym) was developed, an elaboration on this. For details see www.idef.com/default.html. 


\subsection{The Domain}

The UK Breast Screening Program is a national service that involves a number of screening clinics, each with two or more radiologists. Initial screening tests are by mammography, where one or more X-ray films (mammograms) are taken by a radiographer. Each mammogram is then examined for evidence of abnormality an experienced radiologist (Williams et al. 1998). A decision is then made on whether to recall a patient for further tests because there is suspicion of cancer (Alberdi et al. 2003). Within the screening process it is desirable to achieve the minimum number of false positives (FPs), so that fewer women are recalled for further tests unnecessarily, and the maximum true positive (TP) rate, so that few cancers will be missed (Williams et al. 1998). Unfortunately the radiologists' task is a difficult one because the small number of cancers hidden among a large number of normal cases. The traditional solution for reducing reading errors is to let a peer do double readings. This obviously increases workload. Another solution that is being explored is the use of computer-based image analysis techniques to enable a single radiologist to achieve performance that is equivalent or similar to that achieved by double readings (Williams et al. 1998, Boggis et al. 2000). Computer-aided detection systems can provide radiologists with a useful 'second opinion' (Zheng et al. 2002). The case study in this section involves the introduction of such a system as an aid in screening mammograms. When it is used, the radiologist initially views the mammogram and records a recall decision. Then the system marks a digitised version of the X-ray film with 'prompts' that the radiologist should examine. A final decision on a patient's recall is then taken by the human radiologist based on the original decision and the examination of the marked-up X-ray.

\subsection{Analysis}

In this domain the breast cancer screening programme is in itself a barrier. It mitigates the chance that breast cancer that is difficult to treat develops in women. This barrier could be the starting point for our approach. For the sake of brevity however, our analysis starts at the screening procedure, that is a part of the breast cancer screening programme. It is defined as level 0, shown as a simplified SADT diagram in Figure 3. 


\section{Level 0:Screening Procedure}

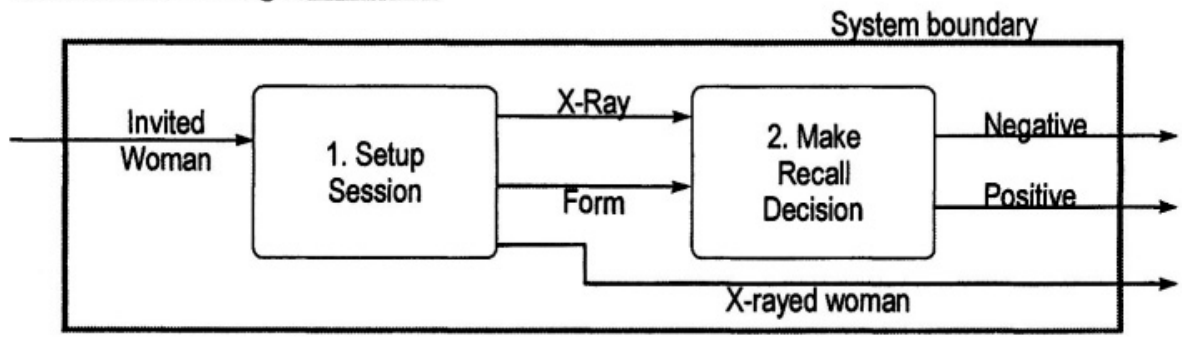

Figure 3. Design of the screening procedure, the top level in this analysis

The screening procedure is a two stage activity. First the session is setup, then the result of this session (an X-Ray and a form with data). In this case, the hazards are identified using a HAZOP (Kletz 1999) procedure, which is not explained in this paper (see Smith and Harrison 2003). Other identification techniques such as forms of task analysis may be used additionally. The results of applying this HAZOP are shown in Table 1.

The next step is to convert the results of the HAZOP into hazards. Further analysis may be required to model the hazards more precisely in terms of causal elements. This can occur for instance by reusing hazard models identified in other systems, or by using expert knowledge.

In this case we further analyse ref. 1.b and 2.b in table 1. Ref. 2.b is used to illustrate how our approach is used in facilitating decision making, while ref 1.b demonstrates how barriers are implemented via the form and embodiment level in different parts of a system.

Table 1. Hazop applied at level 0. Ref. 1.b and 2.b are used in the examples in the text

\begin{tabular}{|c|c|c|c|c|}
\hline $\operatorname{Ref}$ & Item & Guideword & Cause & Consequence \\
\hline 1.a & $\begin{array}{l}\text { Setup } \\
\text { Session }\end{array}$ & More & Too many invited women & $\begin{array}{l}\text { Overload of treatment } \\
\text { system }\end{array}$ \\
\hline 1.b & $\begin{array}{l}\text { Setup } \\
\text { Session }\end{array}$ & Wrong & $\begin{array}{l}\text { X-rays and screening } \\
\text { forms not matched }\end{array}$ & Decision will be faulty \\
\hline 1.c & $\begin{array}{l}\text { Setup } \\
\text { Session }\end{array}$ & Late & $\begin{array}{l}\text { Not ready when radiologist } \\
\text { ready for viewing }\end{array}$ & $\begin{array}{l}\text { Woman will be waiting too } \\
\text { long; Performance problems }\end{array}$ \\
\hline 2.a & $\begin{array}{l}\text { Make } \\
\text { recall } \\
\text { decision }\end{array}$ & Omit & Radiologist error & $\begin{array}{l}\text { No decision, woman will be } \\
\text { waiting too long }\end{array}$ \\
\hline 2.b & $\begin{array}{l}\text { Make } \\
\text { Recall } \\
\text { Decision }\end{array}$ & Wrong & $\begin{array}{l}\text { Something went wrong in } \\
\text { decision; at this level we } \\
\text { cannot determine what. }\end{array}$ & $\begin{array}{l}\text { Possible False Positive or } \\
\text { False Negative }\end{array}$ \\
\hline 2.c & $\begin{array}{l}\text { Make } \\
\text { Recall } \\
\text { Decision }\end{array}$ & Late & $\begin{array}{l}\text { Slow processing; at this } \\
\text { level we cannot determine } \\
\text { why. }\end{array}$ & $\begin{array}{l}\text { Woman will be waiting too } \\
\text { long; Performance problems }\end{array}$ \\
\hline 2.d & $\begin{array}{l}\text { Make } \\
\text { Recall }\end{array}$ & More & $\begin{array}{l}\text { Something went wrong in } \\
\text { decision; at this level we }\end{array}$ & $\begin{array}{l}\text { Confusion in down stream } \\
\text { activities; possible }\end{array}$ \\
\hline
\end{tabular}




\subsection{Barrier based design decisions}

The cause consequence pair indicated by ref $2 . b$ in Table 1 identifies a hazard with two effects: false positives and false negatives (compare Figure 2). It is a primary hazard, as one of these effects directly affects the woman. A false positive requires recalling the woman. The risk here is clearly unacceptable, as a false recall decision has adverse consequences to women, for example stress, and may occur frequently. Hence, a barrier must be designed. This occurs at level 1 , which models the recall decision. In principle, the only activity at this level is to make the decision. The barrier is an additional element. The corresponding IDEF diagram is shown in Figure 4 , the decision being element 1.1, the barrier 1.2.

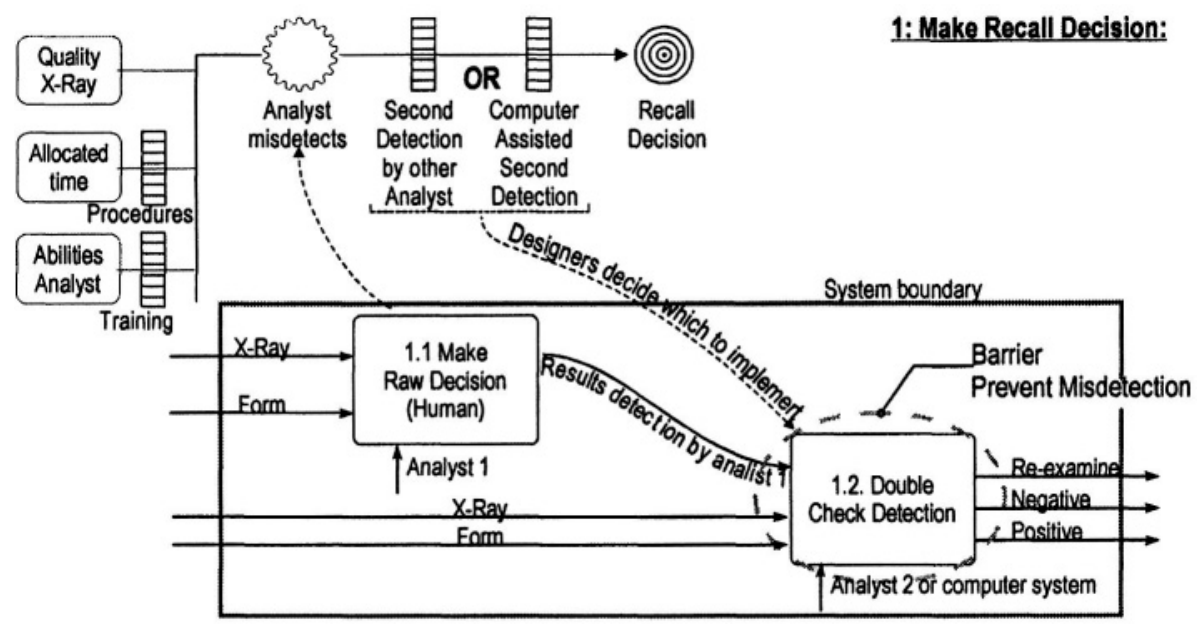

Figure 4. An analyst may make an error when detecting. Hence, system designers must decide on which barrier to use, a second reading by another analyst, or computer assisted prompting 
2.2 alternative A: Double Check Decision by Human Agent:

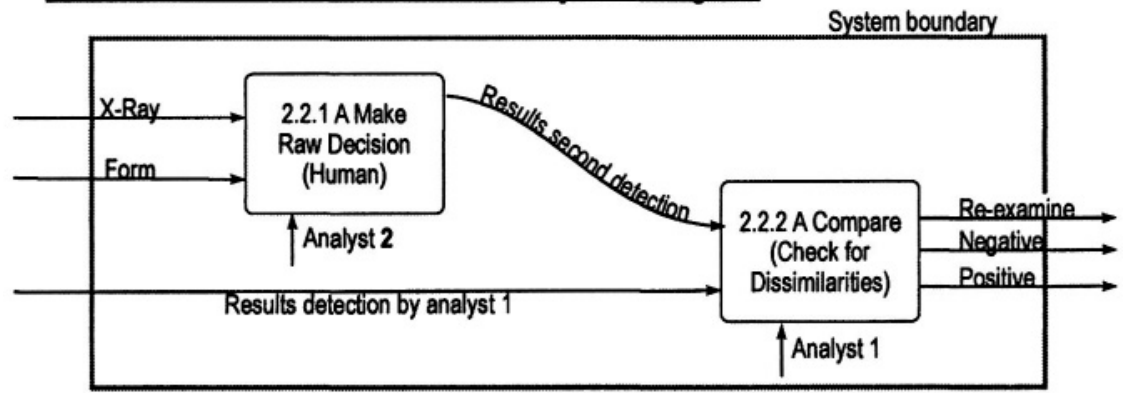

2.2 alternative B: Double check decision assisted by computer system:

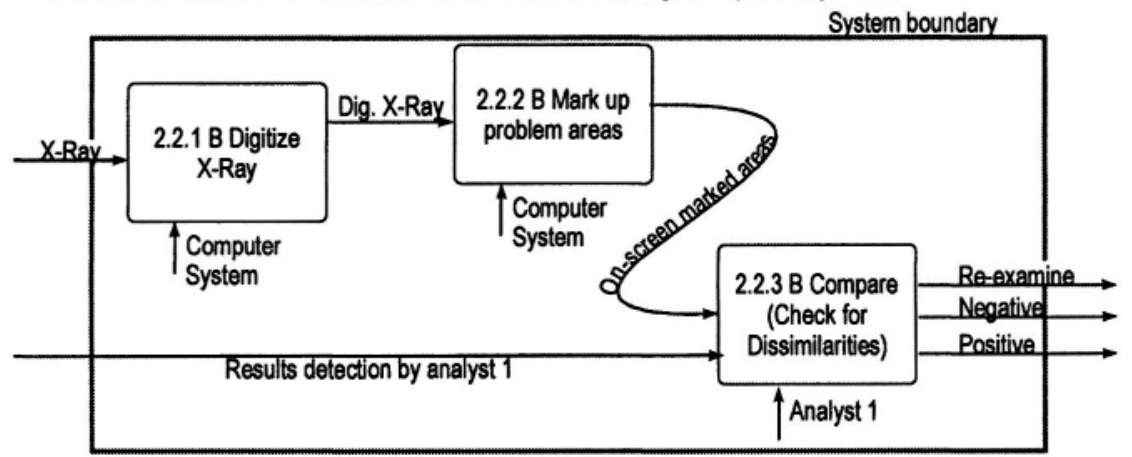

Figure 5. Form level design of Double check barrier: Alternative A: using a human agent; alternative $\mathrm{B}$ using the computer aided detection technique

Two alternative solutions for a barrier that defend against misdetection by the analyst are conceivable: (a) A double check of the recall decision by a human agent, and (b) a recall decision assisted by the novel computer system. The first solution has two activities. A second analyst repeats the detection of the first analyst, thus providing a second opinion. The first analyst then compares this to his own, making use of notes on problem areas provided by the second analyst. The second solution functions differently. After the analyst completed an initial marking, a computer system prompts detected problem areas. The analyst then compares these to the original marking. Both alternative solutions are shown in Figure 5.

The solutions can be analysed in terms of functional hazards. Typical design questions may be which functional hazards may disable each alternative and which new functional and primary hazards are created by these each option. Many of these hazards are caused by human factors, and can be found with traditional human factor analysis methodologies such as task analysis, a process which is not further discussed here. The result of this analysis is shown in Table 2. 
Table 2. Hazards created by the two alternative barriers for double checking the raw decision

\begin{tabular}{|c|c|c|c|}
\hline \multicolumn{2}{|c|}{ Alternative A: Human agent } & \multicolumn{2}{|c|}{ Alternative B: Computer System } \\
\hline Functional Hazards & $\begin{array}{l}\text { New hazards to } \\
\text { other systems }\end{array}$ & Functional Hazards & $\begin{array}{l}\text { New hazards to } \\
\text { other systems }\end{array}$ \\
\hline $\begin{array}{l}\text { FH A.1: Analyst } 2 \\
\text { makes same mistake } \\
\text { as analyst } 1 \\
\text { (e.g. because } \\
\text { relatively few } \\
\text { positives occur) }\end{array}$ & $\begin{array}{l}\text { FH A.4: Analyst } 1 \\
\text { overly relies on } \\
\text { analyst } 2 \text {. (the B/C } \\
\text { screening decision } \\
\text { is directly affected } \\
\text { by this) }\end{array}$ & $\begin{array}{l}\text { FH B.1: Misdetection } \\
\text { because of poor } \\
\text { software }\end{array}$ & $\begin{array}{l}\text { FH B.4: Analyst } \\
\text { overly relies on } \\
\text { computers system. } \\
\text { (the B/C screening } \\
\text { decision is directly } \\
\text { affected by this) }\end{array}$ \\
\hline $\begin{array}{l}\text { FH A.2: Analyst } 1 \\
\text { ignores second } \\
\text { detection }\end{array}$ & $\begin{array}{l}\text { FH A.5: Human } \\
\text { resources used up }\end{array}$ & $\begin{array}{l}\text { FH B.2: Analyst } \\
\text { ignores computer } \\
\text { system } \\
\text { FH B.3: Analyst }\end{array}$ & \\
\hline FH A.3: & & oversees & \\
\hline Communication Error & & $\begin{array}{l}\text { dissimilarities in } \\
\text { mark-up. }\end{array}$ & \\
\hline
\end{tabular}

Based on this analysis, the system designers will now decide which alternative to use. The key to this decision is determining which hazards can best be prevented, protected against, or mitigated. Thus identifying which option yields the lowest risk, when barrier effectiveness, costs and tradeoffs are taken into account. In this case it is difficult to mitigate FH A.1 (see Table 2); the analysts carry out the same activity, and thus may produce the same mistake. Also, alternative A can cause performance problems as it uses more human resources. Alternative B however may also create a hazard that is difficult to overcome, FH B.1, poor software. If software development, testing and training is adequate, alternative $\mathrm{B}$ is favourable, as the other functional hazards are similar to those associated with alternative $\mathrm{A}$, or easier to overcome.

To carry out this barrier analysis, SML diagrams are drawn, as shown for alternative A in Figure 6. This displays the H-T relations. Now, barriers can be added, such as adding a procedure to prevent the first analyst from ignores the second analysts' detection, as shown. It might appear much more difficult to find a barrier between other $\mathrm{H}-\mathrm{T}$ pairs however, such as creating a barrier between FH A.1 and T2.2. 


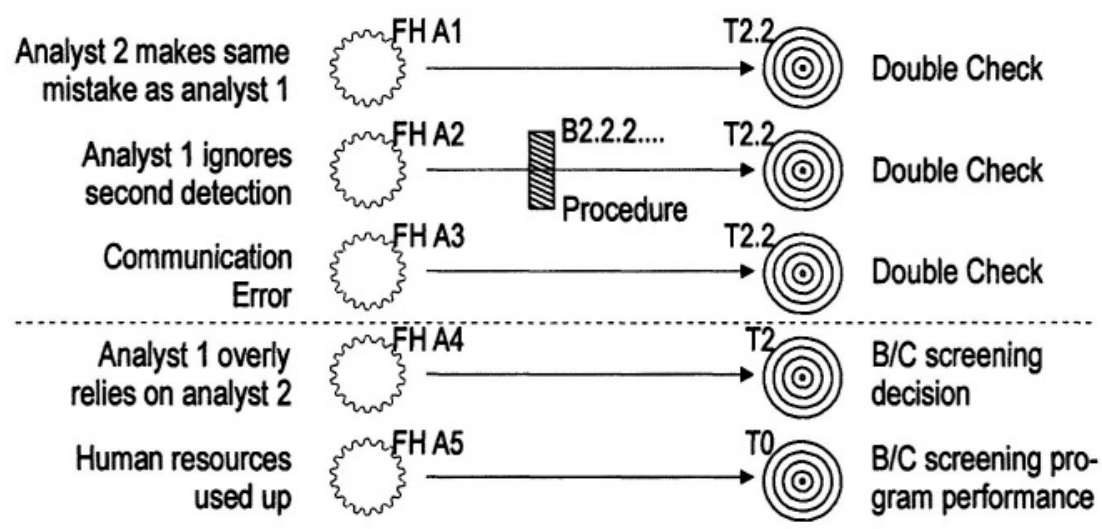

Figure 6. Hazard-Target relations relating to alternative A with one potential barrier shown

\subsection{Designing Barrier Implementation}

Our example now continues with ref 1.b in Table 1, which is used to demonstrate the implementation of a barrier. The hazard exists that either the $\mathrm{X}$-ray or the form are accidentally swapped with that of another woman. This may be due to a human error, for instance a mistake, or it may have another technical cause. The hazard in Figure 7 is due to the simultaneous presence of multiple X-rays photographs, forms and the subsequent processing.

The effect of jumbling forms and X-rays affects the recall decision, but its cause originates earlier in the process, during the initial session. The designers of that session might be aware of this but can only solve it within that context. By analysing the problem using the SML representation instead of focussing at specific parts of the design, at embodiment level, the designers will become better able to avoid an ad-hoc solution. With this overview of the system as a whole they can now decide to use the application of barcodes to both the form and the X-ray and a subsequent check by the computer system which does the mark-up as the barrier.

At form level this barrier is comprised of two parts, (i) the procedure in which the barcodes are applied, and (ii) the subsequent check. These parts determine how the barrier must be embodied in the system. It is not embodied as an isolated part, but in multiple system components; for instance a unique ID is assigned to each women during invitation, applying the barcodes during the X-ray session, and carrying out the check during the computerized mark-up. The barrier is implemented in two ways: as new activities and as controls on existing activities. 


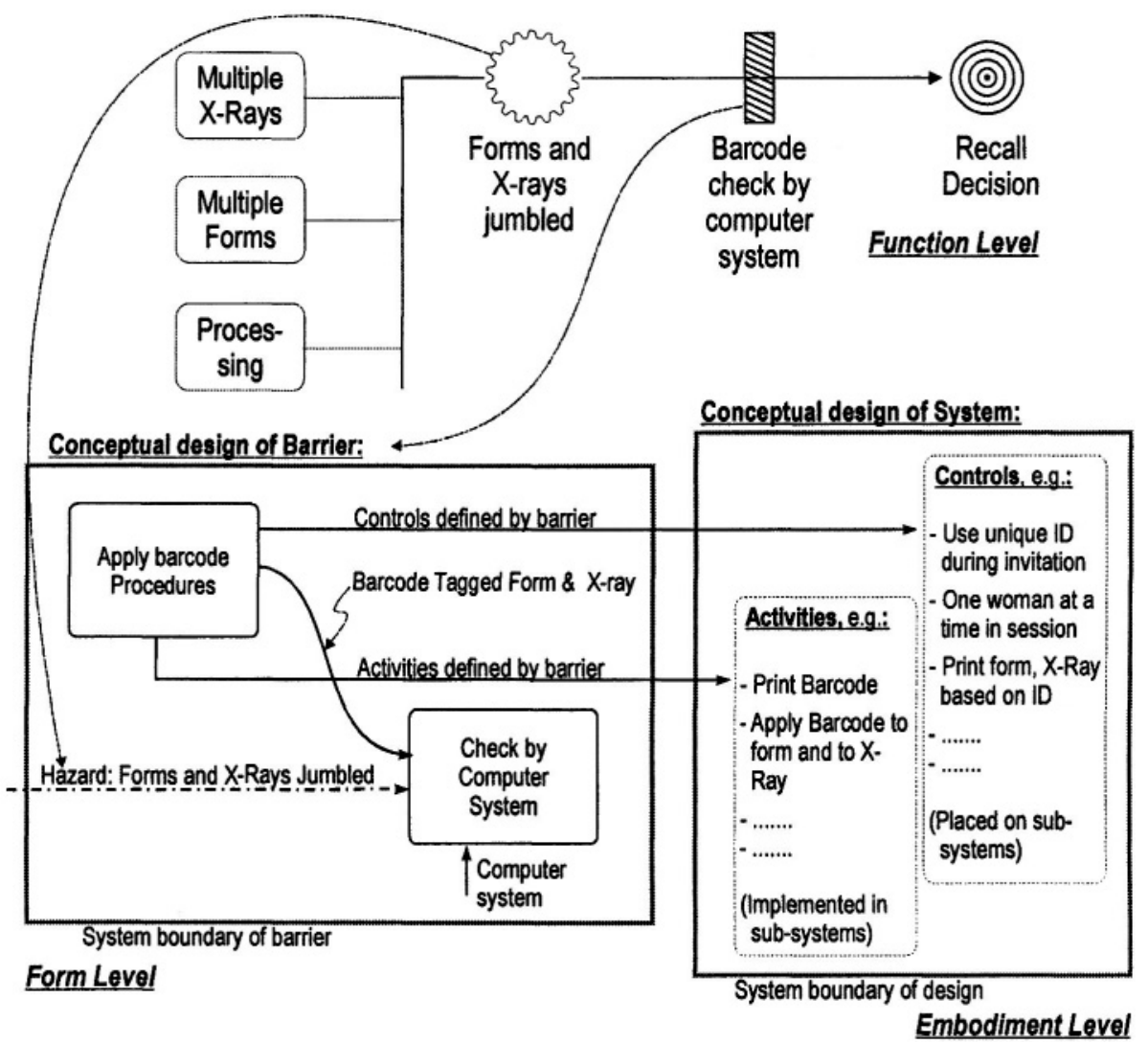

Figure 7. A barrier that defends against jumbling of forms and X-rays. It is represented differently at three different levels: The function, form and embodiment level

Another interesting aspect of this approach is that different barriers may share components at the embodiment level. The computer system was introduced as a barrier to provide the second opinion, however now parts of it have also become part of the new barrier that avoids jumbling of the forms. If a system is subsequently changed, persistent mapping to SML thus prevents inadvertent elimination of a barrier.

\section{CONCLUDING REMARKS}

In this paper a new approach was outlined. The SML helps to structure risk reduction, thus facilitating its design. The conceptual design of barriers and of their embodiment further contributes to this. It was shown how the design of the human role as part of the system is facilitated as well, by not 
directly applying human factors methods to the design of the system, but to the conceptual design of safety and barriers.

Our research will continue on the relation between humans and barriers, and on further extending the vocabulary of the SML and its mappings to systems. One of the disadvantages of the method is that the diagrams used sometimes become quite complex. Therefore, a computer based tool is being developed that helps to manage this information. Also, such a tool will allow for ready reuse and dissemination of diagrams by storing them in a database. The approach can then be used to for instance to measure failure frequencies or operating costs of barriers to facilitate future decision making in design.

\section{ACKNOWLEDGEMENTS}

The collaboration between Delft and York on this subject would not have been possible without funding by the European Union as part of the Advises research training network, GR/N 006R02527. This work was also partly supported by the UK EPSRC DIRC project, GR/N13999. The authors also wish to thank Michael Harrison for his helpful comments.

\section{REFERENCES}

Hollnagel, E. (1993) Human Reliability Analysis : Context and Control. Computers and People Series. London; San Diego, CA: Academic Press. xxvi, 326 p.p.

Arthur D. Little Inc. (2001), American Institute of Chemical Engineers. Center for Waste Reduction Technologies, and American Institute of Chemical Engineers. Center for Chemical Process Safety, Making Ehs an Integral Part of Process Design. New York: CWRT CCPS, American Institute of Chemical Engineers. xvi, 164 p.p.

Schupp, B.A., S.M. Lemkowitz, L.H.J. Goossens, A.R. Hale, and H.J. Pasman. (2002) Modeling Safety in a Distributed Technology Management Environment for More CostEffective Conceptual Design of Chemical Process Plants. In Computer-Aided Chemical Engineering; European Symposium on Computer Aided Process Engineering - 12. ELSEVIER SCIENCE BV: p. 337-42.

Kirwan, B. (1994) A Guide to Practical Human Reliability Assessment. Bristol, PA: Taylor \& Francis. p.p. 592.

Williams, J. (1986) Heart - a Proposed Method for Assessing and Reducing Human Error, 9th Advances in Reliability Technology Symposium.University of Bradford.

Swuste, P. (1996) Occupational Hazards, Risks and Solutions, thesis, Delft University of technology, $217 \mathrm{p}$.

Harms-Ringdahl, L. (2003), Assessing Safety Functions - Results from a Case Study at an Industrial Workplace. Safety Science, 41(8): p. 701-20.

Schupp, B.A., S.M.L. Lemkowitz, and H.J. Pasman. (2001) Application of the HazardBarrier-Target (Hbt) Model for More Effective Design for Safety in a Computer-Based Technology Management Environment; CCPS ICW: Making Process Safety Pay: the business case, AIChE/CCPS. 
Kecklund, L.J., A. Edland, P. Wedin, and O. Svenson, (1996) Safety Barrier Function Analysis in a Process Industry: A Nuclear Power Application. International Journal of Industrial Ergonomics. 17(3): p. 275-84.

Johnson, W.G. (1980) Mart Safety Assurance Systems. New York: Marcel Dekkerp.

American Institute of Chemical Engineers. (1993) Center for Chemical Process Safety., Guidelines for Safe Automation of Chemical Processes. New York, N.Y.: Center for Chemical Process Safety of the American Institute of Chemical Engineers. xxiv, 424p.

Hollnagel, E. (1999) Accidents and Barriers; Lez Valenciennes. Presses Universitaires de Valenciennes: p. 175-82.

Dowell, A.M. (1998) Layer of Protection Analysis for Determining Safety Integrity Level. Isa Transactions,. 37(3): p. 155-65.

Haddon jr., W. (1973) Energy Damage and the Ten Countermeasure Strategies. Human Factors, 15(4): p. 355-66.

Svenson, O. (1991) The Accident Evolution and Barrier Function (Aeb) Model Applied to Incident Analysis in the Processing Industries. Risk Analysis. 11(3): p. 499-507.

Reason, J.T. (1990) Human Error. Cambridge: Cambridge University Press. 302p.

Williams, L.J., M. Hartswood, and R.J. Prescott (1998) Methodological Issues in Mammography Double Reading Studies. Journal of Medical Screening, 5(4): p. 202-6.

Alberdi, E., A. Povyakalo, L. Strigini, and P. Ayton (2003) Does Incorrect Computer Prompting Affect Human Decision Making? A Case Study in Mammography. In International Congress Series; Proceedings Cars 2003: Computer Assisted Radiology and Surgery. ELSEVIER SCIENCE BV, Amsterdam: p. 938-43.

Boggis, C.R. and S.M. Astley (2000) Computer-Assisted Mammographic Imaging. Breast Cancer Research. 2(6): p. 392-5.

Zheng, B., R. Shah, L. Wallace, C. Hakim, M.A. Ganott, and D. Gur (2002), Computer-Aided Detection in Mammography: An Assessment of Performance on Current and Prior Images. Academic Radiology. 9(11): p. 1245-50.

Kletz, T.A. (1999) Hazop and Hazan Identifying and Assessing Process Industry Hazards. 4th ed. Philadelphia, PA: Taylor \& Francis, xi, 232 p.p.

Smith, S.P. and M.D. Harrison (2003). Reuse in Hazard Analysis: Identification and Support. In Computer Safety, Reliability, and Security, LNCS 2788; SAFECOMP. Springer: p. 382-95. 